\title{
Generalised Beatty sets
}

\author{
Marc Technau \\ Institute of Analysis and Number Theory \\ Graz University of Technology, Kopernikusgasse 24, 8010 Graz, Austria \\ e-mail: mtechnau@math.tugraz.at
}

Received: 2 August 2018

Revised: 10 April 2019

Accepted: 12 April 2019

\begin{abstract}
Generalised Beatty sets, that is, sets of the form $\left\{\left\lfloor m \alpha_{1}+n \alpha_{2}+\beta\right\rfloor: m, n \in \mathbb{N}\right\}$, are studied, where $\lfloor\xi\rfloor$ denotes the largest integer less than or equal to $\xi$. Such sets are shown to be contained in a suitable ordinary Beatty set $\{\lfloor n \alpha+\beta\rfloor: n \in \mathbb{N}\}$ and equal said set save for finitely many exceptions. Moreover, bounds for the largest such exception are given.
\end{abstract}

Keywords: Beatty sequence, Beatty set.

2010 Mathematics Subject Classification: Primary 11B83; Secondary 11K60.

\section{Introduction}

Given a real number $\alpha \geq 1$ and a nonnegative real number $\beta$, the associated (inhomogeneous) Beatty set (or Beatty sequence) is defined by

$$
\mathcal{B}(\alpha, \beta)=\{\lfloor n \alpha+\beta\rfloor: n \in \mathbb{N}\},
$$

where $\lfloor\xi\rfloor$ denotes the largest integer less than or equal to $\xi$. Clearly, consecutive elements of a Beatty sequence differ only by either $\lfloor\alpha\rfloor$ or $\lfloor\alpha\rfloor+1$ and rules for predicting which difference occurs at what position in the sequence were first studied by Johann III Bernoulli [4] with the intent of extrapolating the lunar position whilst—for saving effort—working with approximations to the actually measured speed of revolution, yet still controlling the accumulative error.

Number theoretical interest in Beatty sequences appears to have arisen with the work of Elwin Bruno Christoffel $[5,6]$, who used studied them as a means to coming to terms with the notion of irrationality-a concept which was under scrutiny in these days.

Beatty sequences gained more widespread popularity due to Samuel Beatty [2,3] proposing the following problem for solution in The American Mathematical Monthly: for irrational $\alpha>1$, 
show that $\mathbb{N}$ is a disjoint union of $\mathcal{B}(\alpha, 0)$ and $\mathcal{B}(1 /(1-1 / \alpha), 0)$. Although this cute result appears to be due to John William Strutt ( $3^{\text {rd }}$ Baron Rayleigh) [11], the sequences in question now bear Beatty's name.

The interested reader is invited to trace the references in, e.g., [1] for a (still limited) panorama of the many properties of Beatty sets that have been explored in the literature.

The motivation for the present investigation comes from the work of Steuding and the author [10], wherein, using a result of Vaughan [12], an upper bound for the least prime number in Beatty sets $\mathcal{B}(\alpha, \beta)$ with irrational slope $\alpha$ is obtained. The result may be compared with Linnik's theorem and the resulting bound depends on Diophantine properties of $\alpha$, namely the size of the convergents of its regular continued fraction expansion.

The intent of obtaining similar-yet stronger-results may lead one to replace the Beatty set $\mathcal{B}\left(\alpha_{1}, \alpha_{2}+\beta\right)$ under consideration by the larger set

$$
\mathcal{B}(\boldsymbol{\alpha}, \beta)=\left\{\left\lfloor m \alpha_{1}+n \alpha_{2}+\beta\right\rfloor: m, n \in \mathbb{N}\right\},
$$

where $\boldsymbol{\alpha}=\left(\alpha_{1}, \alpha_{2}\right)$ is a real vector with coordinates $\geq 1$.

For instance, a quick calculation gives

$$
\mathcal{B}((\sqrt{2}, \pi), 0)=\{4,5,7,8,9,10,11,12,13,14,15,16, \ldots\},
$$

and one might eagerly note that lots of primes show up here. However, after calculating even more elements, one cannot help but suspect that

$$
\mathcal{B}((\sqrt{2}, \pi), 0)=\mathbb{N} \backslash\{1,2,3,6\} .
$$

In fact, upon noting that $\mathbb{N}=\mathcal{B}(1,0)$ is a Beatty set, the above equation takes the form

$$
\mathcal{B}(\boldsymbol{\alpha}, \beta)=\{\text { some Beatty set }\} \backslash\{\text { finite set of exceptions }\} .
$$

Perhaps surprisingly, this is the true nature of things here and the findings of this article may be described as follows:

1. We show that (1.2) always holds.

2. We exhibit a suitable choice for the Beatty set on the right hand side of (1.2).

3. We give bounds for the largest exception in (1.2).

None of the proofs are particularly difficult; they split in essentially two cases. First, when $\alpha_{1} / \alpha_{2}$ is irrational, basic distribution properties of the sequence $n \alpha_{1} / \alpha_{2} \bmod 1, n=1,2, \ldots$, are used. Second, when $\alpha_{1} / \alpha_{2}$ is rational some quick calculations and an appeal to the finiteness of the Frobenius number finish the argument.

The plan of the paper is as follows. First, we state our results in Section 2. The corresponding proofs are divided-according to (ir)rationality of $\alpha_{1} / \alpha_{2}$-into Section 3 and Section 4 respectively. Finally, in Section 5 we discuss further directions for future research. 


\section{Statement of results}

A set $\mathscr{X} \subseteq \mathbb{Z}$ is said to be cofinite with respect to $\mathscr{Y} \subseteq \mathbb{Z}$ if $\mathscr{X} \subseteq \mathscr{Y}$ and the set $\mathscr{X}^{\mathrm{c}}=\mathscr{Y} \backslash \mathscr{X}$ is finite. In this case we write $\operatorname{cosup}_{\mathscr{Y}} \mathscr{X}$ for the supremum of $\mathscr{X}^{\mathrm{c}}$. In particular, $\operatorname{cosup}_{\mathscr{Y}} \mathscr{Y}=-\infty$. If we drop the reference to $\mathscr{Y}$, then it is understood that $\mathscr{Y}=\mathbb{N}$.

Here and throughout, $\boldsymbol{\alpha}$ always denotes a vector $\left(\alpha_{1}, \alpha_{2}\right)$ with real coordinates $\geq 1$.

\subsection{Irrational $\alpha_{1} / \alpha_{2}$}

Theorem 2.1. Let $\alpha_{1}, \alpha_{2} \geq 1$ and $\beta \geq 0$ be real numbers and suppose that the quotient $\alpha_{2} / \alpha_{1}$ is irrational. Then $\mathcal{B}(\boldsymbol{\alpha}, \beta)$ is cofinite.

To state a bound on $\operatorname{cosup} \mathcal{B}(\boldsymbol{\alpha}, \beta)$, we need some vocabulary: for a real number $\alpha \geq 1$ write

$$
\alpha=\left[x_{0} ; x_{1}, x_{2}, \ldots\right]=x_{0}+\frac{1}{x_{1}+\frac{1}{x_{2}+\frac{1}{\ddots}}}
$$

for its (regular) continued fraction expansion. If $\alpha$ is irrational, then this expansion has infinitely many terms and the so-called partial quotients $x_{j} \in \mathbb{N}(j=0,1,2, \ldots)$ are uniquely determined. Any finite cutoff

$$
\left[x_{0} ; x_{1}, x_{2}, \ldots, x_{r}\right]
$$

may be written as a reduced fraction $\frac{a}{q}$, the $r$-th convergent to $\alpha$. (Mind that we start with the 0 -th convergent.) For more background on continued fractions the reader is referred to [7,9].

Proposition 2.2. Assume the hypotheses of Theorem 2.1, and let $\frac{a}{q}$ be the $r$-th convergent to $\alpha_{2} / \alpha_{1}$, where

$$
r=\min \left\{x \in \mathbb{Z}: x \geq 1+\log \left(2 \alpha_{1}\right) / \log G\right\}
$$

and $G=\frac{1}{2}(1+\sqrt{5})$ is the Golden ratio. Then

$$
\operatorname{cosup} \mathcal{B}(\boldsymbol{\alpha}, \beta)<\alpha_{1}+q \alpha_{2}+\beta \text {. }
$$

To illustrate this bound, we note that it can be used to prove (1.1) which was previously stated in the introduction without proof. Indeed, a calculation reveals that $r=4$ in (2.2) and

$$
\pi / \sqrt{2}=[2 ; 4,1,1,15, \ldots] .
$$

This leads to the fraction $\frac{a}{q}=[2 ; 4,1,1,15]=\frac{311}{140}$. Thus,

$$
\operatorname{cosup} \mathcal{B}((\sqrt{2}, \pi), 0)<\sqrt{2}+140 \pi \approx 441.2,
$$

and a calculation of all small elements in $\mathcal{B}((\sqrt{2}, \pi), 0)$ now establishes $(1.1)$. We note that when reversing the roles of $\pi$ and $\sqrt{2}$ (which incidentally does not change the associated Beatty set) one obtains the bound

$$
\operatorname{cosup} \mathcal{B}((\pi, \sqrt{2}), 0)<\pi+311 \sqrt{2} \approx 442.962 .
$$




\subsection{Rational coordinates}

If $\boldsymbol{\alpha}$ has rational coordinates, then $\mathcal{B}(\boldsymbol{\alpha}, \beta)$ can be described quite concisely:

Theorem 2.3. Let $\alpha_{1}=\frac{a_{1}}{q_{1}}$ and $\alpha_{2}=\frac{a_{2}}{q_{2}}$ be reduced fractions with $\alpha_{1}, \alpha_{2} \geq 1$. Furthermore, let $\beta$ be a non-negative real number and put

$$
\tilde{\alpha}=\frac{c}{q_{1} q_{2}}, \quad \tilde{\beta}=\alpha_{1}+\alpha_{2}-\tilde{\alpha}+\beta,
$$

where $c$ denotes the greatest common divisor of $a_{1} q_{2}$ and $a_{2} q_{1}$. Then $\mathcal{B}(\boldsymbol{\alpha}, \beta)$ is cofinite with respect to $\mathcal{B}(\tilde{\alpha}, \tilde{\beta})$.

Note that Beatty sets $\mathcal{B}(\tilde{\alpha}, \tilde{\beta})$ with rational slope $\tilde{\alpha}$ can be written as a union of arithmetic progressions. Moreover, we have a simple analogue of Proposition 2.2:

Corollary 2.4. On the hypotheses of Theorem 2.3 , the set $\mathcal{B}(\boldsymbol{\alpha}, \beta)$ is cofinite if and only if

$$
c \leq q_{1} q_{2}
$$

Moreover, if (2.4) holds, then $\operatorname{cosup} \mathcal{B}(\boldsymbol{\alpha}, \beta) \leq\left\lfloor a_{1} a_{2} / c+\beta\right\rfloor$.

\subsection{The residual case}

For the sake of giving a complete treatment of all possible cases we also include the following theorem, although it does not differ greatly from the previous one.

Theorem 2.5. Let $\alpha_{1}>1$ be irrational and $\alpha_{2}=\frac{a}{q} \alpha_{1}$ with some reduced fraction $\frac{a}{q}$. Furthermore, let $\beta$ be a non-negative real number and put

$$
\tilde{\alpha}=\frac{\alpha_{1}}{q}=\frac{\alpha_{2}}{a}, \quad \tilde{\beta}=\tilde{\alpha}+\beta .
$$

Then $\mathcal{B}(\boldsymbol{\alpha}, \beta)$ is cofinite with respect to $\mathcal{B}(\tilde{\alpha}, \tilde{\beta})$.

A result corresponding to Corollary 2.4 is also immediate.

\section{Proof of the results for irrational $\alpha_{1} / \alpha_{2}$}

Before turning to the details, we sketch the underlying idea. We note that $\mathcal{B}(\boldsymbol{\alpha}, \beta)$ is a union of ordinary Beatty sets, namely

$$
\mathcal{B}(\boldsymbol{\alpha}, \beta)=\bigcup_{n \geq 1} \mathcal{B}\left(\alpha_{1}, n \alpha_{2}+\beta\right)
$$

By Lemma 3.2 membership of $x \in \mathbb{Z}$ to each of these Beatty sets is determined by the fractional part of $x / \alpha_{k}(k=1,2)$ belonging to a certain interval modulo one and $x$ not being too small. On varying $n$, these 'detector intervals' can be shown to eventually cover $\mathbb{R} / \mathbb{Z}$ (here the assumption that $\alpha_{1} / \alpha_{2}$ be irrational enters the picture) and, consequently, there is a finite collection of Beatty 

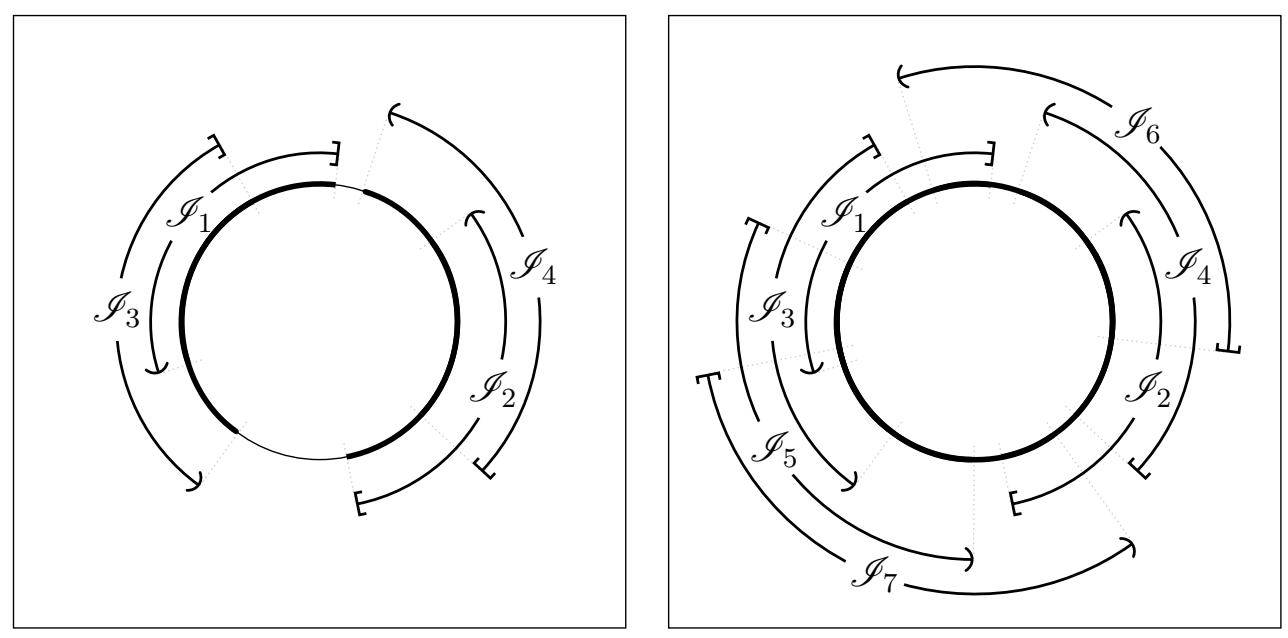

Figure 1. Intervals $\mathscr{I}_{n}$ modulo one (identified with arcs on a circle and lifted up for the sake of readability) 'detecting' elements of $\mathcal{B}(\pi, n \sqrt{2})(n=1,2,3,4)$. When drawing the picture for $n=1,2, \ldots, 7$, the full circle is covered.

sets $\mathcal{B}\left(\alpha_{1}, n \alpha_{2}+\beta\right)$ such that every sufficiently large integer $x$ belongs to at least one element of said collection. The reader is referred to Fig. 1, which illustrates this point for the generalised Beatty set in (1.1).

Suppose that $\alpha$ has the continued fraction expansion (2.1) and let $\frac{a}{q}$ be the $r$-th convergent to $\alpha$. Then

$$
|q \alpha-a| \leq q^{-1}
$$

The size of $a$ and $q$ depends, of course, on the values of $x_{0}, \ldots, x_{r}$. However, via comparison with the continued fraction expansion of the Golden ratio,

$$
G=\frac{1}{2}(1+\sqrt{5})=[1 ; 1,1, \ldots],
$$

and using Binet's formula, a lower bound in terms of $r$ can be given (see, e.g., [10] for the details).

Lemma 3.1. Let $\frac{a}{q}$ be the $r$-th convergent to some real number $\alpha>0$. Then $q \geq G^{r-1}$, where $G$ is given by (3.3).

The following lemma is certainly well-known; it gives a convenient test for membership in a Beatty set.

Lemma 3.2. An integer $x$ is contained in the Beatty set $\mathcal{B}(\alpha, \beta)$ if and only if $x>\alpha+\beta-1$ and $x \alpha^{-1} \in\left[\frac{\beta-1}{\alpha}, \frac{\beta}{\alpha}\right) \bmod 1$.

We shall require some very basic distribution properties of the sequence $(n \alpha)_{n}$ modulo one; of course, much more is true (see, e.g., [8]), but the following simple lemma suffices for our purpose.

Lemma 3.3. Let $\alpha$ be a real number and suppose that $\frac{a}{q}$ is a reduced fraction satisfying (3.2). Then

$$
\sup _{\xi \in[0,1)} \min _{n \leq q}\{\xi-n \alpha\} \leq 2 q^{-1}
$$

where $\{\xi\}=\xi-\lfloor\xi\rfloor$ denotes the fractional part of $\xi$. 
Proof. This is immediate from the triangle inequality.

Proof of Theorem 2.1. Let $x$ be large, $x>x_{0}$ say, where the value of $x_{0}$ is yet to be determined. We intend to show that $x \in \mathcal{B}(\boldsymbol{\alpha}, \beta)$. By (3.1) it suffices to exhibit some $n$ such that $x \in$ $\mathcal{B}\left(\alpha_{1}, n \alpha_{2}+\beta\right)$. In view of Lemma 3.2, this is equivalent to

$$
\begin{gathered}
x \alpha_{1}^{-1}-n \alpha_{1}^{-1} \alpha_{2} \in\left[\frac{\beta-1}{\alpha_{1}}, \frac{\beta}{\alpha_{1}}\right) \bmod 1, \\
n<\left(x+1-\beta-\alpha_{1}\right) \alpha_{2}^{-1} .
\end{gathered}
$$

Now let $\frac{a}{q}$ be the $r$-th convergent to $\alpha=\alpha_{1}^{-1} \alpha_{2}$. By taking $r$ sufficiently large, we may assume that

$$
q>2 \alpha_{1} .
$$

Then Lemma 3.3 ensures the existence of some $n \leq q$ satisfying (3.4). The theorem now follows by choosing $x_{0}$ such that the right hand side of (3.5) is larger than $q$.

To give a proof of Proposition 2.2, a closer inspection of the above proof suffices: on applying Lemma 3.1, we find that (3.6) is satisfied if

$$
1+\log \left(2 \alpha_{1}\right) / \log G \leq r
$$

Since $\operatorname{cosup} \mathcal{B}(\boldsymbol{\alpha}, \beta) \leq x_{0}$, we arrive at (2.3) after determining an admissible value for $x_{0}$ in the way outlined above.

Remark 3.1. Of course, given some $\alpha$ for which the growth of the denominators of its $r$-th convergents as $r \rightarrow \infty$ is known, Proposition 2.2 may be replaced with some smaller quantity.

\section{The case when $\alpha_{1} / \alpha_{2}$ is rational}

As a motivation recall that given an integer vector $\boldsymbol{x} \in \mathbb{Z}^{r}(r \geq 2)$ with positive coprime coordinates, one has

$$
\mathbb{Z} x_{1}+\ldots+\mathbb{Z} x_{r}=\mathbb{Z}
$$

When one replaces $\mathbb{Z}$ with $\mathbb{N}_{0}$, the set of non-negative integers, on the left hand side, then every sufficiently large integer on the right hand side can still be represented. The largest integer that cannot be thus represented is called the Frobenius number of $\boldsymbol{x}$ and shall be denoted by $g(\boldsymbol{x})$, i.e.,

$$
g(\boldsymbol{x})=\operatorname{cosup}_{\mathbb{N}_{0}}\left(\mathbb{N}_{0} x_{1}+\ldots+\mathbb{N}_{0} x_{r}\right) .
$$

If $r=2$, then one has the simple formula

$$
g\left(x_{1}, x_{2}\right)=x_{1} x_{2}-x_{1}-x_{2} .
$$

However, to fit our convention that Beatty sets are generated via multiplication by positive integers, we shall work with

$$
g_{+}\left(x_{1}, x_{2}\right)=\operatorname{cosup}\left(\mathbb{N} x_{1}+\mathbb{N} x_{2}\right)=x_{1} x_{2} .
$$


Now looking at a Beatty set $\mathcal{B}(\boldsymbol{x}, \beta)$, one finds that

$$
\mathcal{B}(\boldsymbol{x}, \beta) \supseteq\left\{n \in \mathbb{N}: n>g_{+}\left(x_{1}, x_{2}\right)+\beta\right\} .
$$

It is a straightforward matter to extend this argument:

Proof of Theorem 2.3. For positive integers $m, n$ write

$$
m \frac{a_{1}}{q_{1}}+n \frac{a_{2}}{q_{2}}=\left(m \frac{a_{1} q_{2}}{c}+n \frac{a_{2} q_{1}}{c}\right) \frac{c}{q_{1} q_{2}} .
$$

This already proves the inclusion $\mathcal{B}(\alpha, \beta) \subseteq \mathcal{B}(\tilde{\alpha}, \tilde{\beta})$. Since the expression in the parentheses in (4.2) can be made to equal any integer

$$
>g_{+}\left(a_{1} q_{2} c^{-1}, a_{2} q_{1} c^{-1}\right)=\ell \quad(\text { say })
$$

if the positive integers $m$ and $n$ are chosen appropriately, we have

$$
\operatorname{cosup}_{\mathcal{B}(\tilde{\alpha}, \tilde{\beta})} \mathcal{B}(\alpha, \beta) \leq\left\lfloor\frac{\ell c}{q_{1} q_{2}}+\beta\right\rfloor=\left\lfloor\frac{a_{1} a_{2}}{c}+\beta\right\rfloor .
$$

This proves the theorem.

Proof of Corollary 2.4. This is a trivial consequence of Theorem 2.3, and the fact that $\mathcal{B}(\tilde{\alpha}, \tilde{\beta})$ is cofinite if and only if $\tilde{\alpha} \leq 1$; the stated bound is obtained from (4.3) upon noting that $\mathcal{B}(\tilde{\alpha}, \tilde{\beta})=$ $\{x \in \mathbb{N}: x>\alpha+\tilde{\beta}-1\}$ if (2.4) holds.

In the setting of Corollary 2.4, one may naively suspect that the inequality given in (4.3) may in fact be an equality. However, this is not generally the case. Indeed, when $\tilde{\alpha} \leq \frac{1}{2}$, the problem of determining $\operatorname{cosup} \mathcal{B}(\boldsymbol{\alpha}, \beta)$ is related to the position of large gaps (with respect to $\tilde{\alpha}$ ) in

$$
\mathbb{N} a_{1} q_{2} c^{-1}+\mathbb{N} a_{2} q_{1} c^{-1}
$$

To illustrate this point, consider the generalised Beatty set

$$
\mathscr{B}=\mathcal{B}\left(\left(\frac{3}{2}, 2\right), 0\right)=\{3,5,6,7,8, \ldots\}=\mathbb{N} \backslash\{1,2,4\} .
$$

Here (4.4) takes the form

$$
\mathbb{N} 3+\mathbb{N} 4=\mathbb{N} \backslash\left\{1,2,3,4,5,6,{ }_{-} 8,9,{ }_{-}-12\right\}
$$

and we have $\tilde{\alpha}=\frac{1}{2}$. Note that $g_{+}(4,3)=12$, yet $\operatorname{cosup} \mathscr{B}<6=\lfloor 12 \tilde{\alpha}\rfloor$, because $\lfloor 12 \tilde{\alpha}\rfloor=\lfloor 13 \tilde{\alpha}\rfloor$ and 13 is an element of $\mathbb{N} 3+\mathbb{N} 4$. However,

$$
\operatorname{cosup} \mathscr{B}=4=\lfloor 8 \tilde{\alpha}\rfloor=\lfloor 9 \tilde{\alpha}\rfloor .
$$

Proof of Theorem 2.5. Simply write

$$
m \alpha_{1}+n \frac{a}{q} \alpha_{1}=(m q+n a) \frac{\alpha_{1}}{q}
$$

and argue as in the proof of Theorem 2.3. 


\section{Open problems}

We start by noting that Proposition 2.2 only gave $\operatorname{cosup} \mathcal{B}((\sqrt{2}, \pi), 0) \leq 441$, whereas in actuality $\operatorname{cosup} \mathcal{B}((\sqrt{2}, \pi), 0)=6$ (see (1.1)). In general, we suspect that our bounds on the exceptional set in (1.2) are quite poor. (Here it is understood that the shift in the Beatty set on the right hand side of (1.2) is chosen as to have as few members in the exceptional set as possible.) In fact, our understanding of the exceptional set is even far from optimal in the case when considering generalised Beatty sets $\mathcal{B}(\boldsymbol{\alpha}, \beta)$ with rational quotient $\alpha_{1} / \alpha_{2}$, as showcased by our discussion of small gaps in Section 4.

Therefore, for arbitrary generalised Beatty sets $\mathcal{B}(\boldsymbol{\alpha}, \beta)$, we list the following two problems:

Problem 5.1. Obtain better bounds for the largest member of the exceptional set in (1.2).

Problem 5.2. Investigate structural properties of the exceptional set in (1.2).

Here the last problem is intentionally vague in its formulation. A rather more specific but related question was posed by one of the anonymous referees:

Problem 5.3. Obtain non-trivial bounds for the smallest member of the exceptional set in (1.2).

\section{Acknowledgements}

This work is part of the author's doctoral dissertation at Würzburg University. The author gratefully acknowledges the encouragement of his advisor, Jörn Steuding. Financial support by both Würzburg University and Graz Technical University is greatly appreciated.

\section{References}

[1] Banks, W. D., \& Shparlinski, I. E. (2009) Prime numbers with Beatty sequences, Colloq. Math., 115, 2, 147-157.

[2] Beatty, S. (1926) Problem 3173, Amer. Math. Monthly, 33, 159.

[3] Beatty, S., \& Ostrowski, A., \& Hyslop, J., \& Aitken, A. C. (1927) Solutions to problem 3173, Amer. Math. Monthly, 34, 159-160.

[4] Bernoulli, J. III (1772) Sur une nouvelle espece de calcul, In Recueil pour les Astronomes, volume I, 255-284, Berlin.

[5] Christoffel, E. B. (1873) Observatio arithmetica, Annali di Mat. (2), 6, 148-153.

[6] Christoffel, E. B. (1887) Lehrsätze über arithmetische Eigenschaften der Irrationalzahlen, Annali di Mat. (2), 15, 253-276.

[7] Hardy, G. H., \& Wright, E. M. (2008) An introduction to the theory of numbers, sixth edition, Oxford University Press, Oxford. 
[8] Kuipers, L., \& Niederreiter, H. (1974) Uniform distribution of sequences, John Wiley \& Sons, New York.

[9] Perron, O. (1954) Die Lehre von den Kettenbrüchen. Band I, third edition, B.G. Teubner Verlagsgesellschaft, Leipzig.

[10] Steuding, J., \& Technau, M. (2016) The least prime number in a Beatty sequence, J. Number Theory, 169, 144-159.

[11] Strutt, J. W. (1926) The Theory of Sound, second edition, Macmillan, London.

[12] Vaughan, R. C. (1978) On the distribution of $\alpha p$ modulo 1, Mathematika, 24, 135-141. 